Liu, C., González, V.A., Liu, J., Rybkowski, Z.K., Schöttle, A., Álvarez, C.M., and Pavez, I. 2020.

"Accelerating the LPS Uptake using Virtual Reality and Serious Games: A Socio-technical Conceptual Framework." In: Tommelein, I.D. and Daniel, E. (eds.). Proc. $28^{\text {th }}$ Annual Conference of the International Group for Lean Construction (IGLC28), Berkeley, California, USA, doi.org/10.24928/2020/0058, online at iglc.net.

\title{
ACCELERATING THE LAST PLANNER SYSTEM® (LPS) UPTAKE USING VIRTUAL REALITY AND SERIOUS GAMES: A SOCIO- TECHNICAL CONCEPTUAL FRAMEWORK
}

\author{
Canlong Liu', Vicente A. González², Jiamou Liu ${ }^{3}$, Zofia Rybkowski, \\ Annett Schöttle ${ }^{5}$, Claudio Mourgues Álvarez ${ }^{6}$, and Ignacio Pavez
}

\begin{abstract}
The Last Planner System ${ }^{\circledR}$ (LPS) use in projects faces both social and technical challenges associated with the nature of its planning and control structure, and the way it is implemented on-site. Thus, the social dynamics inherent with the LPS implementation within construction organisations should be shaped appropriately to harmonically match its technical components and vice versa. Although recent research has revealed that a successful implementation of the LPS is highly dependent upon how its social and technical aspects are managed and interrelated, there are still problems with the LPS implementation in the design phase and on-site. Some simulation games have been used for LPS training, but they do not seem to meet the demands of the industry to accelerate LPS adoption. This paper finds that Virtual Reality (VR) and Serious Games (SG) could be more effective in spreading LPS by mitigating critical barriers than conventional approaches. We also discuss how the capabilities of VR/SG technology can assist in tackling some of LPS adoption barriers that relate back to the social dynamics that are engendered and the technical knowledge that is required to implement the LPS smoothly. We also propose a conceptual framework to study the socio-technical dynamics of the LPS and identified six questions to be addressed in future research.
\end{abstract}

\section{KEYWORDS}

Socio-technical Dynamics, Virtual Reality Serious Game, Last Planner ${ }^{\circledR}$ System, Language Action Perspectives, Collaboration.

1 PhD Student, Department of Civil and Environmental Engineering, The University of Auckland, Auckland, New Zealand, cliu324@aucklanduni.ac.nz, orcid.org/0000-0001-6653-9920

$2 \mathrm{PhD}$, Senior Lecturer, Department of Civil and Environmental Engineering, The University of Auckland, Auckland, New Zealand, v.gonzalez@auckland.ac.nz, orcid.org/0000-0003-3408-3863

3 PhD, Senior Lecturer, Department of Computer Science, The University of Auckland, Auckland, New Zealand,jiamou.liu@auckland.ac.nz, orcid.org/0000-0002-0824-0899

$4 \quad \mathrm{PhD}$, Associate Professor, Department of Construction Science of the College of Architecture, Texas A\&M University, Texas, USA, zrybkowski@tamu.edu, orcid.org/0000-0002-0683-5004

5 Senior Consultant, Refine Projects AG, Stuttgart Area, Germany, annett.schoettle@refine.team, orcid.org/0000-0001-6001-7320

$6 \mathrm{PhD}$, Associate Professor, Dept. of Construction Engineering and Management, Pontificia Universidad Católica de Chile, Santiago, Chile, cmourgue@ing.puc.cl, orcid.org/0000-0001-6706-2255

$7 \mathrm{PhD}$, Assistant Professor, School of Business and Economics, Universidad del Desarrollo, Santiago, Chile, ignaciopavez@udd.cl,orcid.org/0000-0001-5257-5330 


\section{INTRODUCTION}

The benefits of Last Planner System ${ }^{\circledR}$ (LPS) have been well-reported, and its popularity has grown in time, but a holistic implementation is still difficult in many organisations (Fernandez-Solis et al. 2013; Perez and Ghosh, 2018). Other studies on LPS have realised that this challenge originates from the lack of capacity of the project team and organisation to adapt processes and behaviours to both the social and technical nature of LPS (González et al. 2015b; Perez and Ghosh 2018; Schöttle and Nesensohn 2019). These studies support the notion that the limitations in understanding that LPS is a lean methodology that encompasses not only technical expertise and knowledge associated with planning and control but also it engenders changes in the social dynamics of construction organisations. As it turns out, many studies reported that these aspects of LPS are not managed effectively, imposing a slow LPS uptake/diffusion and may remain unsolved in time (Fuemana et al. 2013; Hunt and Gonzalez 2018; Stevens 2014). Therefore, overcoming LPS limitations and barriers of adoption is an urgent need for a construction industry willing to fully embrace lean construction ideas (Fernandez-Solis et al. 2013). Some simulation games, including digital and hands-on games, have been widely used for training and simulation of LPS implementation, but they do not seem to meet the demands of the industry to accelerate LPS adoption (González et al. 2015a).

Virtual Reality (VR) and Serious Games (SG) technologies have the potential to shift behaviour, engender positive attitudes of users towards specific goals, enhance selfefficacy, improve technical knowledge acquisition, among other features. VR and SG can also enable virtual social environments where actual individuals interact, behaviour can be observed in "quasi-realistic" environments, and targeted education and training can be provided (Feng et al. 2018; Gao et al. 2019; Lovreglio et al. 2018). This paper will conceptually discuss simulation and training framework using VR and SG to tackle LPS implementation barriers, building on the understanding that LPS is a socio-technical system that enables the adoption of lean principles in construction organisations along with generating particular socio-technical dynamics. In the following sections, existing literature associated with this research is examined, and a conceptual framework for further research is proposed.

\section{LITERATURE REVIEW}

\section{CRITICAL BARRIERS WHEN IMPLEMENTING LPS}

The shift from traditional organisations towards lean organisations results in a higher level of organisational integration, decentralised decision-making structures, flexible communication channels, and a flow centred operating system (González et al. 2015b). However, users might have poor ability to adapt the transition from a traditional organisational culture to a lean construction culture due to the fragmented and complex nature of the construction industry (Poshdar et al. 2019). LPS implementation barriers can be characterised as socially-driven and production-driven involving the technical nuances of a production process, but also involving the knowledge required to manage the issue associated with processes (González et al. 2015b).

In this paper, we have renamed the production-driven aspect of LPS as technicallydriven to relate it back to knowledge-based challenges. As shown in Table 1, The authors further explore barriers to LPS implementation. Table 1 categorises barriers in both the

social and technical domains. Each category has several critical barriers and influence factors that relate to those critical barriers. 
In the social domain, the most frequently reported barrier is a fear of transition to lean organisations because stakeholders are more accustomed to using a conventional production control methodologies (Alarcón et al. 2005). There are some reasons why project participants are reluctant to use the LPS, including ignoring the subcontractors' opinions when making decisions on-site, unwillingness to learn new technical knowledge (Poshdar et al. 2019). El-Sabek et al. (2018) studied the challenges of LPS implementation in international megaprojects (IMPs). They identified that organisational inertia and lack of trust are the most common barriers found in LPS implementation, especially if the team members come from different cultural backgrounds. Additionally, other studies suggested that these problems have contributed to an unwillingness to make changes to the traditional way individuals used to behave (e.g. independently manage the process and make the plan) (Poshdar et al. 2019). In table 1, we classified these problems to factors that possibly affect $B 1$ : Resistance to change, which could be one of the critical barriers in the social domain. Porwal et al. (2010) focused on social barriers at the team or project level, including poor team chemistry, lack of stable commitments and low trust. Daniel et al. (2015) conducted an empirical study of procurement on LPS implementation and noting that the lack of subcontractor's involvement could be a barrier for a successful LPS implementation. These barriers are regarded as influence factors associated with $B 2$ : Lack of collaboration.

Table 1: Socio-technical barriers when implementing LPS

\begin{tabular}{|c|c|c|c|}
\hline Domains & Critical barriers & Influence Factors & References \\
\hline \multirow{6}{*}{ Social } & \multirow{3}{*}{$\begin{array}{c}\text { B1: Resistance to } \\
\text { change }\end{array}$} & Lack of trust & \multirow{3}{*}{$\begin{array}{c}\text { (El-Sabek and } \\
\text { McCabe, 2018); } \\
\text { (Poshdar et al. 2019) }\end{array}$} \\
\hline & & $\begin{array}{l}\text { Ignore subcontractors' opinion } \\
\text { when making decisions on site }\end{array}$ & \\
\hline & & $\begin{array}{l}\text { Passionately subscribed to } \\
\text { conventional production control } \\
\text { methodology }\end{array}$ & \\
\hline & \multirow{3}{*}{$\begin{array}{l}\text { B2: Lack of } \\
\text { cooperation }\end{array}$} & Poor team chemistry & \multirow{3}{*}{$\begin{array}{l}\text { (Porwal et al. 2010); } \\
\text { (Daniel et al. 2015) }\end{array}$} \\
\hline & & $\begin{array}{l}\text { Lack of commitment to LPS } \\
\text { implementation }\end{array}$ & \\
\hline & & $\begin{array}{l}\text { Minimum involvement of } \\
\text { construction subcontractors }\end{array}$ & \\
\hline \multirow{3}{*}{ Technical } & \multirow{3}{*}{$\begin{array}{l}\text { B3: Lack of } \\
\text { understanding of } \\
\text { LPS principles } \\
\text { and training } \\
\text { methods }\end{array}$} & $\begin{array}{l}\text { Lack of implementation } \\
\text { Knowledge }\end{array}$ & \multirow{3}{*}{$\begin{array}{c}\text { (Porwal et al. 2010) } \\
\text { (Fernandez-Solis et } \\
\text { al. 2013) } \\
\text { (Hamzeh 2009) }\end{array}$} \\
\hline & & $\begin{array}{l}\text { Learning component of the } \\
\text { methodology remains at a basic } \\
\text { level }\end{array}$ & \\
\hline & & Misunderstanding of guidelines & \\
\hline
\end{tabular}

In the technical domain, most of the studies emphasise human capital problems associated with lack of staff training and teaching methods, lack of implementation knowledge, and misunderstandings about guidelines (Hamzeh 2009), because they are too busy to spare time in improving learning (Porwal et al. 2010). Besides, current pedagogical methods are not able to effectively convey the theory behind the LPS because of its complexity, consequently posing a negative impact on its adoption and execution (Fernandez-Solis et al. 2013). In the technical aspect, these barriers are considered as influence factors 
associated with knowledge-based challenges. Some studies argued that these technical factors hinder more effective dissemination and uptake of lean knowledge into the construction industry (Fuemana et al. 2013; González et al. 2015b; Hunt and Gonzalez 2018). In this regard, as shown in Table 1, we relate these factors to B3: Lack of understanding of LPS principles and training methods, which is considered as a critical barrier in the technical domain.

\section{SOCIO-TECHNICAL DYNAMICS IN LPS IMPLEMENTATION}

From a social standpoint, LPS focuses on controlling the production system by managing conversations, relationships, and commitments among participants (González et al. 2015b). Essentially, it facilitates elaborating not only practical plans and reliable workflows but also building project teams aimed at increasing the accountability of its members (González et al. 2015b; Schöttle and Nesensohn 2019).

Social dynamics refers to understanding the relationship between individual-level interactions and group-level behaviours by exploring responses resulting from interactions among internal individuals (González et al. 2015b). Hence, individuals are affected by the social behaviour of others (Durlauf and Young 2004). Therefore, the industry needs to have a better understanding of the interplay between social dynamics and lean thinking in construction organisation to investigate the most critical social elements (González et al. 2015b).

Existing research reveals how the implementation of the LPS changes social elements in the construction project, and how influencing these social elements to facilitate LPS implementation (Pavez and González 2012). Moreover, (González et al. 2015b) shed light on the interplay between LPS practices and social dynamics variables such as goal setting, trust and power distance (centralised or decentralised) in construction. They emphasised that a flat organisational structure (low power distance) could generate a high degree of trust among individuals and facilitate the integration of the team. Also, specific, challenging and achievable goals seem to motivate the team because they could strengthen mutual trust by stressing collaboration and commitment (González et al. $2015 b$ ). Furthermore, collaboration, reliability of commitment, and trust are fundamental social dynamics between subcontractors and contractors or when implementing the LPS (e.g. Priven and Sacks 2013).

Therefore, when relating back to critical implementation barriers, we note that continuously implementing LPS and training adds value to socio-technical aspects associated with acquiring knowledge and improving the social dynamics, thereby addressing its socio-technical barriers. We also assume that a successful LPS training not only depends on technical dynamics (e.g. improvement of LPS knowledge and work method) but also requires efficient management of social dynamics (e.g. encourage collaboration and change). Therefore, in this paper, these dynamics with LPS implementation are regarded as socio-technical dynamics. Although some research provides theoretical guidelines for implementing LPS, the underlying mechanisms and conceptualisation need further empirical investigation and test.

\section{Simulation And Training APPRoAches of LPS}

Simulation and training have been applied in industry to mitigate these socio-technical barriers. Conventional LPS implementation and training are operated in workshops or meetings during construction, which requires lean consultants to teach LPS principles directly and encourage participants to follow lean steps based on paper-based instructions 
or templets (Hackett et al. 2019). For example, project participants are required to post stickers with task details on whiteboards to engage in pull planning and root cause analysis. This hands-on method could reinforce reflective learning and mutual trust since people are personally involved in a realistic project but may not be efficient with respect to knowledge dissemination, because it is normally adopted along with a real project, which requires subcontractors to meet together physically. Also, it does not seem to be a flexible experimental platform with an editable environment that enables research of socio-technical dynamics.

Simulation games offer alternative means to address barriers related to knowledge dissemination and cooperation by presenting lean principles and introducing management approaches (González et al. 2014). Hands-on games are widely applied in LPS training. These games simulate both conventional management approach and a lean-based approach by asking participants to assemble $\mathrm{Lego}^{\mathrm{TM}}$ pieces, for example, to form a schematic house. Essentially, participants repeatedly go through and compare these two principles to highlight the value that LPS brings, along with delivering lean knowledge. In some cases, the benefits of multiskilling of the workforce and the pull concept are highlighted in the LEAPCON experiment (Sacks et al. 2007), but they are not widely used in training. Following that, LPS-based Villego game is released as a commercial product, aiming to teach LPS principles through two round games in a day (Villego 2014), which has been the most prominent training approach in the industry. Another example of LPS educational practice is the LEBSCO, which is a comprehensive LPS simulation game offering a quick training session in the classroom context. These hands-on simulation games, especially Villego ${ }^{\circledR}$ are engaging and accessible enough to attract participants to participate and deliver knowledge in a short time, but they still have limitations such as space restriction and simplified tasks. For example, (1) in some circumstance, teams cannot co-locate to train in LPS together in a cross-national project, (2) make too many simplifications that may limit the users' expectancy and learning.

Digital forms of simulation games could offer advantages of economy, flexibility and accessibility. It would allow participants to develop an understanding of LPS process without space restriction barriers. Also, the digital medium enables computer simulation in details and have educational functions. However, existing digital game such as "The Parade of Trade Game" are abstract in expression, and mechanistically simple, but lacking in realism. Therefore, there is a need to explore a hybrid method that has advantages of both traditional coaching and simulation games but has the potential to overcome constraints such as space restriction, simplified tasks, abstract and timeconsuming.

\section{Social Network Analysis And Language-Action Perspectives: THE UNDERSTANDING THE SOCIO-TECHNICAL NATURE OF LPS}

The Social Network (SN) is a social structure to present the relationships between agents (e.g. individuals or organisations), including all patterns of social agents (organisations or individuals), dyadic ties (relationships between two agents), and social interactions (Castillo et al. 2018). In order to characterise the social structure, Social Network Analysis (SNA) presents agents as nodes and ties as lines in terms of network theory. The attributes of interest among agents are reflected by qualitatively evaluating the social networks such as centrality and density of communication channels in this systematic analysis (Priven and Sacks 2013). As a sociological tool, SNA has been applied in several construction cases, which includes two major levels. At the company level, Pryke (2004) 
emphasised that SNA is an essential means to investigate interrelationships within construction companies such as subcontractors and general contractor. For instance, SNA has been used to explain different patterns of cooperation between transnational corporations and has been successfully associated with the performance of a company. At the project level, SNA has assisted in explaining key behavioural patterns between subcontractors and general contractors (Priven and Sacks 2013). It has been demonstrated that LPS strengthen the social networks among subcontractors.

The Language Action Perspectives (LAP) highlights both what people actually do during communications and how language coordinates the activities. LAP has been used at modelling action workflow or developing the commitment networks in many domains. Based on the LAP and Speech Act Theory, the promise cycle regards every communication unit as a commitment loop, performing by two people with four different phases such as request, negotiation, performance and satisfaction (Fernando Flores 2012). Viana et al. (2017) further embedded this model into the LPS workflow to investigate the communication change in LPS implementation while generating an overview of construction meetings, but the limited amount of data hampers further research. These research show that the SNA/LAP has a promising prospect in studying social dynamics in LPS implementation if the difficulty in data gathering can be mitigated.

\section{VIRTUAL REALITY AND SERIOUS GAME}

Serious Games (SG) have gained significant attention over the last decade being increasingly demanded from the education industry (Laamarti et al. 2014). Most serious games were board games for military training in the early days, but recent SG comes from the software engineering and gaming industry and is understood as video games that have the purpose of education and training beyond entertainment. Connolly et al. (2012) also suggested that SG is designed to educate people and modify their behaviours, highlighting that SG can perform behaviour analysis and training in addition to entertaining people. SG has been primarily used in education and training (Feng et al. 2018). These studies have provided empirical evidence to support that SG could be regarded as a practical training and instructional technique for personal skill development, knowledge acquirement, and team cooperation. One reason is that SG allows players to interact with the models and avatars by providing feedback accordingly to improve knowledge or behaviours (Lovreglio et al. 2018). Another reason is that players can be engaged and better recall the concepts they have learned compared to traditional learning methods (Feng et al. 2018).

In addition to training purposes, SG has the potential to analyse human behaviour by evaluating both their decision-making and responses to controllable stimuli (Lovreglio et al. 2018). Owing to its capability to collect behavioural data, SG can help to explain behavioural motivation, uncover behavioural patterns and modify behaviours. Therefore, the SG platform provides a "Virtual Laboratory" with more experimental controls than traditional tools, demonstrating to enable a better understanding of behavioural patterns and changes than more traditional education (Feng et al. 2018; Gao et al. 2019; Lovreglio et al. 2018).

Virtual Reality (VR) technology has been widely promoted in recent decades within the rapid growth of the smartphone industry, enabling high-quality, economical, and accessible VR experience (LaValle 2017). It can be defined as "a synthetic or virtual

environment which gives a person a sense of reality" (Jayaram et al. 1997). LaValle (2016) further explained that these VR technologies induce targeted behaviours by providing an 
artificially simulated environment while making them exceedingly difficult to distinguish between the real world and the virtual environment, which possibly enable "ecological validity" (consistent behaviours observed in a VR and the real world).

VR and SG technologies have the potential to shift behaviour, engender positive attitudes of users towards specific goals, enhance self-efficacy, and improve technical knowledge acquisition, among other features (Feng et al. 2018). VR and SG can also enable virtual social environments where actual individuals interact, behaviour can be observed in "quasi-realistic" environments, and targeted education and training can be provided (Lovreglio et al. 2018).

Another advantage of combining VR and SG is that the amount of behavioural data that can be collected during the gaming-based experiment is much more than traditional social experimental methods, and can be extracted for review at any time allowing the investigation of communication target, duration, tone, gesture and the gaze players behave when interacting with others (Chittaro and Buttussi 2015). This type of data, which are difficult to collect with conventional experiments, can provide evidence to identify what factors are influencing a behaviour. However, VR and SG have yet significant limitations that need to be addressed. For example, VR and SG users can suffer from motion sickness, headache, and dizziness when they are wearing head-mounted displays, and they can have limited tactile and olfactory (a sensory-enriched) virtual experiences (Feng et al. 2018; LaValle 2016). Furthermore, it is also questionable if VR SG can increase the team building in the virtual world as hands-on training that increases the closeness among team members.

In construction, VR and SG have been adopted in interactive training for off-site production (Goulding et al. 2012). These studies provided comprehensive discussions on the VR and SG potential. So far, there has been no research assessing the combination of VR and SG with the intention of speeding up the uptake of LPS; thus, there is still a need to understand how to design and implement VR and SG to support LPS implementation.

\section{CONCEPTUAL FRAMEWORK}

\section{Linking Problems With Virtual Reality and Serious Games}

As suggested in Table 1, critical barriers can be split into the social domain and technical/pedagogical domain. In the social domain, the lack of cooperation is a pressin problem when starting the LPS implementation. In the technical domain, people have a poor understanding of the LPS's technical intrincacies and only few pedagogical approaches can effectively deliver the related knowledge (González et al. 2015a).

By understanding the advantages of VR and SG (Table 2, Table 3) and the LPS' critical barriers of implementation based on the literature review, Table 4 explores the relevance of VR and SG in solving critical LPS barriers. We proposed a VR SG-based framework (detailed discussed in next section) that can provide a highly immersive and engaging experience in a controllable environment while providing behavioural correction opportunities (related to factor V1 V5 and S1 S5). This helps to identify key behaviours to be shifted towards best LPS practice, and improve knowledge retention through the training and learning framework provided by VR and SG (Lovreglio et al. 2018), having a great potential to mitigate barrier B3. A standardised, repeatable virtual experience with convenient features (related to factors: V1, V2, V3, V5, S2, S4, S5) would allow participants to strengthen cooperation through continuous practice and help the researcher to identify these cooperative behaviours (Feng et al. 2018), thereby 
providing solutions to barriers B2. By enabling people to be in a transparent and collaborative work environment, the application of VR and SG (related to factors: V1, V2, V3, S2, S4, S5) can improve trust among project team members and generate psychological ownership, which could engender a type of "cultural revolution" (Curtis and White 2002), reversing the trend of resistance to change (B1).

Table 2: Advantages of Serious Games

\begin{tabular}{|c|c|c|}
\hline $\begin{array}{c}\text { Advantages of Serious } \\
\text { Games }\end{array}$ & References & Description \\
\hline S1: Reinforce learning & (Lovreglio et al. 2018) & Recall the memory \\
\hline S2: Highly Engaging & (Feng et al. 2018) & Rntertain people \\
\hline S3: Correct human behaviour & (Lovreglio et al. 2018) & Rectify incorrect response \\
\hline $\begin{array}{c}\text { S4: Investigate human } \\
\text { behaviour }\end{array}$ & (Connolly et al. 2012) & $\begin{array}{l}\text { Tracking and recording } \\
\text { decisions and behaviour }\end{array}$ \\
\hline S5: Controlled environment & (Lovreglio et al. 2018) & Storyline can be easily edited \\
\hline
\end{tabular}

Table 3: Advantages of Virtual Reality

\begin{tabular}{|c|c|c|}
\hline Advantages of Virtual Reality & References & Description \\
\hline V1: Immersion & (LaValle 2016) & $\begin{array}{l}\text { Create more "realistic } \\
\text { feelings." }\end{array}$ \\
\hline V2: Realism & (LaValle 2016) & $\begin{array}{c}\text { Simulate visual or interaction } \\
\text { experience }\end{array}$ \\
\hline $\begin{array}{l}\text { V3: Highly engaging and } \\
\text { perception }\end{array}$ & (Feng et al. 2018) & $\begin{array}{l}\text { High emotional and } \\
\text { physiological arousal }\end{array}$ \\
\hline V4: Concretisation of knowledge & (Feng et al. 2018) & $\begin{array}{l}\text { Bypassing abstract symbol } \\
\text { systems to reinforce learning }\end{array}$ \\
\hline V5: Behaviour observation & (Lovreglio et al. 2018) & $\begin{array}{l}\text { Flexible in collecting } \\
\text { behaviour data }\end{array}$ \\
\hline
\end{tabular}

Table 4: Advantages of VR and SG in Solving Critical LPS barriers.

\begin{tabular}{ccccccccccc}
\hline \multirow{2}{*}{ Critical barriers } & \multicolumn{10}{c}{ Advantages of VR and SG } \\
\cline { 2 - 10 } & V1 & V2 & V3 & V4 & V5 & S1 & S2 & S3 & S4 & S5 \\
\hline B1: Resistant to change & $\sqrt{ }$ & $\sqrt{ }$ & $\sqrt{ }$ & & & & $\sqrt{ }$ & $\sqrt{ }$ & $\sqrt{ }$ & $\sqrt{ }$ \\
B2: Lack of cooperation & $\sqrt{ }$ & $\sqrt{ }$ & $\sqrt{ }$ & & $\sqrt{ }$ & & $\sqrt{ }$ & & $\sqrt{ }$ & $\sqrt{ }$ \\
B3: Lack of Understanding & $\sqrt{ }$ & $\sqrt{ }$ & $\sqrt{ }$ & $\sqrt{ }$ & $\sqrt{ }$ & $\sqrt{ }$ & $\sqrt{ }$ & $\sqrt{ }$ & $\sqrt{ }$ & $\sqrt{ }$ \\
\hline
\end{tabular}

\section{Forming THE CONCEPTUAL FRAMEWORK}

In this section, Figure 1 presents the conceptual framework that we proposed in this paper to overcome LPS implementation barriers. Based on insights from the literature review and the discussion of the linkage between problems and VR SG, the logic of this framework (as shown in the left side of figure 2) is to design experiments by defining anticipated behaviours related back to critical barriers (shown as B1, B2, B3), adopt 
technical tools to conduct experiment and observation, use sociological methodologies to explain this simulation process, and finally involve pedological approaches to tackle these barriers. If needed, further observations can be applied to provide interventional strategies that will improve research outcomes.

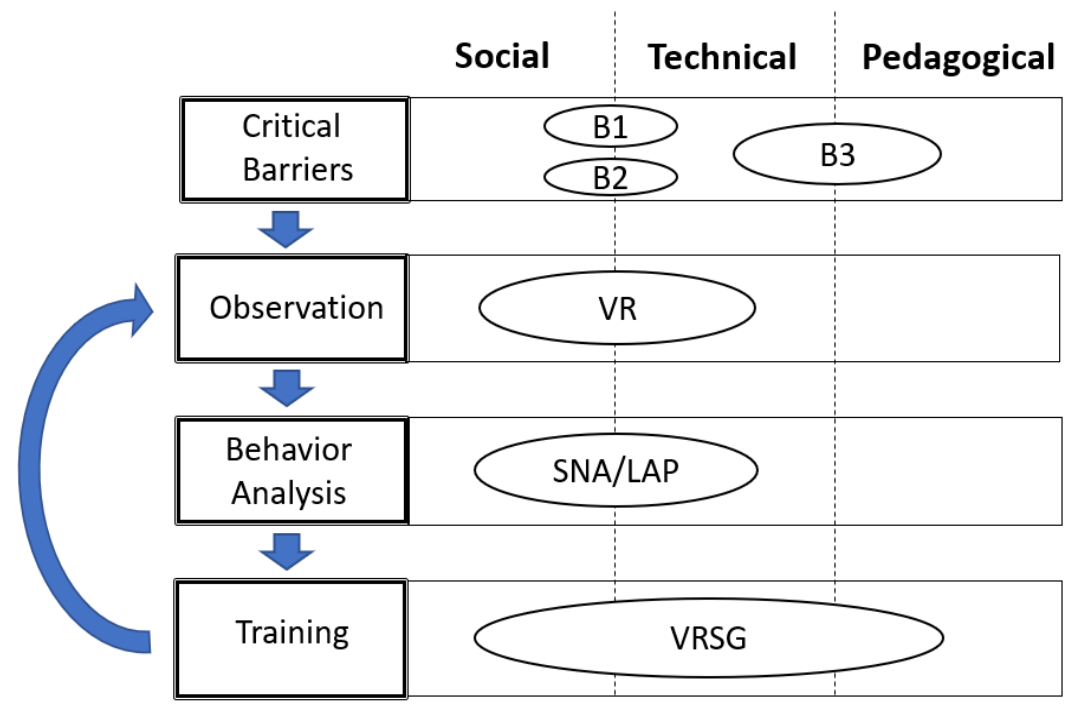

Figure 1: Conceptual framework for studying socio-technical dynamics when implementing the LPS

More specifically, we divided concepts into social, technical and pedagogical dimensions. The right side of Figure 1 explains specific concepts in these domains such as VR, SG, LAP, SNA. VR can not only provide participants with an immersive experience by technically simulating the actual implementation of LPS (planning and control perspectives), but it can also consider the social aspects of implementation as it can provide an environment that can mimic a real construction project (including machinery, equipment, workers). It can also enable fluid communication and interaction between people (e.g. a multi-user environment or social VR). Given the ecological validity or realism of VR, people can behave as similar as they do in the real world. Also, their behavioural responses can be analysed through shifting variables because of editable attributes of VR. The social and technical attributes of VR provide a reliable interface for SNA and LAP to collect behavioural data objectively, while the SNA and LAP can interpret participant behaviour in the macro and micro level with providing conceptualised social explanations, which might provide feedback for optimising the LPS training and education. The integration of VR and SG offers additional attributes that can generate additional educational outcomes compared to VR.

This conceptual framework is illustrated further by way of an example to offer a clear understanding. Firstly, some behavioural patterns related to critical barriers can be conceptualised to set a research goal, such as lack of collaboration, this pattern could provide evidence for the unwillingness to communicate with other people in VR-based scenarios, but VR can only help us observe behaviour without a really depth understanding of the "whys". As a robust behavioural analysis tool. SNA/LAP can identify and explain these patterns underpinned by statistical and sociological bases. For example, the VR-based observation shows some people communicate extensively with a single person, but avoid communication and eye contact with others, which may imply a 
lack of collaboration in the organisation. By using a certain statistical extent through SNA concerning the data of gesture and eye gaze captured by VR, we can identify these barriers. This collaboration trend may change along with repeated training sessions and the change be identified by SNA and VR. According to the outcomes of the training, we can develop some educational strategies to facilitate collaboration, which could serve as the starting point of another round of observations. Note that this is a simple example because many factors can influence collaboration. More research in terms of socio-technical dynamics and VR and SG based LPS training should be conducted in the future.

\section{CONCLUSIONS}

We carried out a literature review on both socio-technical barriers in LPS implementation and their relationship with socio-technical dynamics. The current research and specific concepts such as VR, SG, SNA, and LAP that might be used to tackle critical barriers were extensively explored, highlighting the importance of socio-technical dynamics when people start using LPS. Findings indicate the significance of VR and SG in delivering knowledge by training and studying social dynamics by conducting a behavioural analysis event though they have some limitations in motion sickness and questionable in facilitating team building. As a result, we proposed a conceptual framework for studying socio-technical dynamics for LPS implementation based on a discussion of these concepts. This framework aims to contribute to future applications of VRSG for LPS training and socio-technical dynamics research.

In light of the literature review and conceptual framework, some research questions can be established (1) What behavioural patterns related back to critical socio-technical barriers can be observed in the experiment? (2) How can the socio-technical dynamics in LPS implementation be more comprehensively analysed by using SNA/LAP? (3) Which social dynamics/aspects can significantly improve LPS implementation? (4) What are the essential social elements and intervention strategies that have the potential to be considered to tackle critical barriers? (5) Can LPS be more effectively delivered if VR SG technology is used for its teaching when compared to traditional methods? and (6) How user-friendly and effective is the improved VR SG in terms of LPS training?

The study is limited by a lack of discussion on behaviour recognition and consideration of the validity of VR in a multi-user environment. Further work is required to develop a VR SG prototype and validate it through case studies.

\section{REFERENCES}

Alarcón, L. F., Diethelm, S., Rojo, O., and Calderon, R. (2005). "Assessing the impacts of implementing lean construction." Proc. 13 th Ann Conf. Int. Group for Lean Construction, 387-393.

Castillo, T., Alarcón, L. F., and Salvatierra, J. L. (2018). "Effects of Last Planner System Practices on Social Networks and the Performance of Construction Projects." Journal of Construction Engineering and Management, 144(3).

Chittaro, L., and Buttussi, F. (2015). "Assessing knowledge retention of an immersive serious game vs. A traditional education method in aviation safety." IEEE Transactions on Visualization and Computer Graphics, 21(4), 529-538.

Connolly, T. M., Boyle, E. A., MacArthur, E., Hainey, T., and Boyle, J. M. (2012). "A systematic literature review of empirical evidence on computer games and serious games." Computers and Education, Elsevier Ltd., 59(2), 661-686. 
Curtis, E., and White, P. (2002). "Resistance to change causes and solutions." Nursing Management, The MIT Press, 8(10), 15-20.

Daniel, E. I., Pasquire, C., and Dickens, G. (2015). "Exploring the implementation of the last planner ${ }^{\circledR}$ system through iglc community: Twenty one years of experience." Proc. $23^{\text {rd }}$ Ann Conf. Int. Group for Lean Construction, July, 153-162.

Durlauf, S. N., and Young, H. P. (2004). Social dynamics. Mit Press.

El-Sabek, L. M., and McCabe, B. Y. (2018). "Framework for Managing Integration Challenges of Last Planner System in IMPs.” Journal of Construction Engineering and Management, 144(5), 1-14.

Feng, Z., González, V. A., Amor, R., Lovreglio, R., and Cabrera-Guerrero, G. (2018). "Immersive virtual reality serious games for evacuation training and research: A systematic literature review." Computers and Education, Elsevier, 127, 252-266.

Fernandez-Solis, J. L., Porwal, V., Lavy, S., Shafaat, A., Rybkowski, Z. K., Son, K., and Lagoo, N. (2013). "Survey of motivations, benefits, and implementation challenges of last planner system users." Journal of Construction Engineering and Management, 139(4), 354-360.

Flores, F. (2012). "Instilling a culture of commitment in our working relationships." Conversations For Action and Collected Essays, 15.

Fuemana, J., Puolitaival, T., and Davies, K. (2013). "Last planner system - A step towards improving the productivity of new zealand construction." Proc. $21^{\text {st }}$ Ann Conf. Int. Group for Lean Construction.

Gao, Y., Gonzalez, V. A., and Yiu, T. W. (2019). "The effectiveness of traditional tools and computer-aided technologies for health and safety training in the construction sector: A systematic review." Computers and Education.

González, V.A., Orozco, F., Senior, B., Ingle, J., Forcael, E., and Alarcón, L.F. (2015a). "LEBSCO: Lean-Based Simulation Game for Construction Management Classrooms." Journal of Professional Issues in Engineering Education and Practice, 141(4).

González, V. A., Sacks, R., Pavez, I., Poshdar, M., Alon, L. Ben, and Priven, V. (2015b). "Interplay of lean thinking and social dynamics in construction." Proc. $23^{\text {rd }}$ Ann Conf. Int. Group for Lean Construction, 681-690.

González, V. A., Senior, B., Orozco, F., Alarcon, L. F., Ingle, J., and Best, A. (2014). "Simulating lean production principles in construction: A Last Planner-driven game." Proc. $22^{\text {nd }}$ Ann Conf. Int. Group for Lean Construction, 1221-1232.

Goulding, J., Nadim, W., Petridis, P., and Alshawi, M. (2012). "Construction industry offsite production: A virtual reality interactive training environment prototype." Advanced Engineering Informatics, Elsevier Ltd, 26(1), 103-116.

Hackett, V., Harte, P., and Chendo, J. (2019). "The Development and Use of Last Planner ${ }^{\circledR}$ System (LPS) Guidance." Proc. 27th Ann Conf. Int. Group for Lean Construction, 651-662.

Hamzeh, F. R., and LCI. (2009). "Improving construction workflow-The role of production planning and control." (March).

Hunt, R. J., and Gonzalez, V. A. (2018). "Innovation in the New Zealand construction industry - Diffusion of the Last Planner System." Proc. $26^{\text {th }}$ Ann Conf. Int. Group for Lean Construction, 422-431.

Jayaram, S., Connacher, H. I., and Lyons, K. W. (1997). "Virtual assembly using virtual reality techniques.” CAD Computer Aided Design, 29(8), 575-584.

Laamarti, F., Eid, M., and El Saddik, A. (2014). "An overview of serious games." Int. J. of Computer Games Technology, Hindawi Publishing Corporation, 2014. 
LaValle, S. (2016). "Virtual reality." National Programme on Technology Enhanced Learning (NPTEL).

Lovreglio, R., Gonzalez, V., Feng, Z., Amor, R., Spearpoint, M., Thomas, J., Trotter, M., and Sacks, R. (2018). "Prototyping virtual reality serious games for building earthquake preparedness: The Auckland City Hospital case study." Advanced Engineering Informatics, Elsevier, 38(February), 670-682.

Pavez, I., and González, V. (2012). "Social dynamic of improvement when using the last planner system: A theoretical approach." Proc. $20^{\text {th }}$ Ann Conf. Int. Group for Lean Construction.

Perez, A. M., and Ghosh, S. (2018). "Barriers faced by new-adopter of Last Planner System ${ }^{\circledR}$ : a case study." Engineering, Construction and Architectural Management, 25(9), 1110-1126.

Porwal, V., Fernández-Solís, J., Lavy, S., and Rybkowski, Z. K. (2010). "Last planner system implementation challenges." Proc. $18^{\text {th }}$ Ann Conf. Int. Group for Lean Construction, 548-556.

Poshdar, M., Gonzalez, V. A., Antunes, R., Ghodrati, N., Katebi, M., Valasiuk, S., Alqudah, H., and Talebi, S. (2019). "Diffusion of Lean Construction in Small to Medium-Sized Enterprises of Housing Sector." Proc. $27^{\text {th }}$ Ann Conf. Int. Group for Lean Construction, 383-392.

Priven, V., and Sacks, R. (2013). "Social network development in Last Planner System ${ }^{\mathrm{TM}}$ implementations." Proc. $21^{\text {st }}$ Ann Conf. Int. Group for Lean Construction, 474-485.

Pryke, S. D. (2004). "Analysing construction project coalitions: Exploring the application of social network analysis." Constr. Management and Economics, 22(8), 787-797.

Sacks, R., Esquenazi, A., and Goldin, M. (2007). "LEAPCON: Simulation of lean construction of high-rise apartment buildings." Journal of Construction Engineering and Management, 133(7), 529-539.

Schöttle, A., and Nesensohn, C. (2019). "The Beauty of a Phase-Overlapping Last Planner System ${ }^{\circledR}$ With Incorporated Takt.” Proc. $27^{\text {th }}$ Ann Conf. Int. Group for Lean Construction, 441-450.

Stevens, M. (2014). "Increasing adoption of lean construction by contractors." Proc. $22^{\text {nd }}$ Ann Conf. Int. Group for Lean Construction.

Viana, D. D., Formoso, C. T., and Isatto, E. L. (2017). "Understanding the theory behind the Last Planner System using the Language-Action Perspective: two case studies." Production Planning and Control, Taylor \& Francis, 28(3), 177-189.

Villego 2014. "Villego-last planner simulation." <http://www.villego .com/> (May 8, 2020) 